\title{
LOCAL SYNCHRONIZATION OF CYCLIC COUPLED HYPERCHAOTIC SYSTEMS AND ITS CIRCUIT IMPLEMENTATION
}

\author{
K. S. Ojo \\ Department of Physics \\ University of Lagos \\ Akoka, Lagos \\ Nigeria \\ kaystephe@yahoo.com
}

A. I. Egunjobi
Department of Physics
Moshood Abiola Polytechnic
Abeokuta
Nigeria

\author{
A. O. Adelakun \\ Department of Physics \\ Federal University of Technology \\ Akure \\ Nigeria
}

\author{
E. I. Udoh \\ Department of Physics \\ University of Lagos \\ Akoka, Lagos \\ Nigeria
}

Article history:

Received 22.07.2019, Accepted 22.09.2019

\begin{abstract}
Most of the available research works on cyclic coupling of chaotic systems focussed on either analytical and numerical results or numerical and experimental results. This research paper, investigates synchronization of two cyclic coupled hyperchaotic systems using analytical, numerical and experimental techniques. Based on Routh-Hurwitz criterion, analytical condition for stable synchronization of the hyperchaotic systems are derived. The results obtained from MultiSIM and analog circuit confirm the effectiveness and feasibility of the analytical results. It is worthy of note that the cyclic coupling synchronization scheme gives several synchronization options, save synchronization time and cost. Moreover, cyclic coupling synchronization scheme has potential applications in biological information transmission networks.
\end{abstract}

\section{Key words}

Cyclic coupling, Routh-Hurwitz criterion, Hyperchaotic oscillator, MultiSIM, Analog implementation.

\section{Introduction}

Synchronization phenomenon is one of the most exciting behaviour of coupled nonlinear dynamical systems due to its significant and relevant applications in physical, chemical, biological and complex systems in general [Strogatz, 1994; Alligood et al., 1997; Njah and Ojo, 2010; Ma et al., 2012; Okpabi et al., 2017;
Tong et al., 2016]. Several types of synchronization [Rosenblum et al., 1997; Pecora and Carroll, 1990; Ojo et al., 2016a; Ojo et al., 2011; Ojo et al., 2013b; Gasri et al., 2018], methods of synchronization [Yang, 2012; Njah, 2011; Lu et al., 2013; Ojo et al., 2013a; Adegoke et al., 2013; Yang, 2012], and schemes [Ouannas, 2014; Zhang and Deng, 2014; Dongmo et al., 2018; Ojo et al., 2016b; Yu et al., 2013; Adelakun et al., 2017] have been developed in order to obtain most efficient applications of synchronization to natural and artificial systems. The search for the best coupling techniques to achieve excellent synchronization efficiency has led to the discovery of different coupling techniques such as unidirectional coupling [Adelakun et al., 2014; Rulkov et al., 1995; Ojo et al., 2013c], bidirectional coupling [Kumar et al., 2016; Khan and Poria, 2012], cross coupling [Zhang and Deng, 2014; Mengfei et al., 2015], cyclic coupling [Bera et al., 2016; Olusola et al., 2013; Egunjobi et al., 2018] and others [Liu et al., 2011; Iqbal et al., 2018; Belykh et al., 2005; Kandel et al., 2000]. The types of coupling technique and topology determine the level of stability or instability of synchronization of coupled nonlinear systems.

Having established that several coupling techniques are available for synchronization of nonlinear systems, our particular interest is on analytical, numerical and experimental implementation of synchronization via cyclic coupling technique. The choice of cycling coupling for synchronization is due to the following 
reasons: (i) many synchronization choices (topologies) are available between each pair of variables depending on the dimension of the systems (ii) the available choices (topologies) can be optimized based on your particular objective of synchronization (iii) synchronization cost can be saved based on available choices (topologies)(iv) synchronization time can also be saved based on available choices (topologies) (v) the pair of variables which cannot be synchronization can easily be detected via the analytical criterion. The details of the available choices (topologies) is given in section 3 of this paper.

Cyclic coupled synchronization is a process whereby one system transmits an information to another system using one pair of its variables and then decode the transmitted information via a different pair of variables [Olusola et al., 2013]. It is worthy of note that the cyclic coupling configuration gives a better synchronization results where the diffusive bidirectional coupling configuration failed [Olusola et al., 2013]. Despite several advantages of cyclic coupling synchronization, not many research works have reported in this direction. [Bera et al., 2016; Olusola et al., 2013; Egunjobi et al., 2018; Adelakun et al., 2018].

Investigation on synchronization of cyclic coupled chaotic systems via analytical and numerical simulations was carried out in [Olusola et al., 2013]. It was discovered in the paper that cyclic coupling technique achieves stable synchronization in some chaotic systems where bidirectional mutual coupling technique failed. Also, synchronization of cyclic coupled Sprott systems was investigated and implemented using only electronic experiment [Egunjobi et al., 2018]. To best of our knowledge, synchronization of hyperchaotic systems via cyclic coupling has not been reported. Also, there is no single paper on synchronization of cyclic coupled chaotic systems that combines analytical, numerical and experimental simulations results. Motivated by the above discussion, this research work provides analytical, numerical and experimental evidence of synchronization of cyclic coupled hyperchaotic systems. The choice of hyperchatic system is as result of high complex dynamical behaviour and its attractive application in chaos based secure communication.

This paper is organized as follows. Section 2, provides description of analog circuit for the hyperchaotic system. Section 3, presents the theory of cyclic coupling. Section 4 deals with derivation of synchronization criteria for cyclic coupled hyperchaotic systems. Section 5 deals with results and discussion. Finally, section 6 concludes the paper.

\section{Description of analog circuit for the hyperchaotic system}

The four-dimensional hyperchaotic system proposed in this work can be expressed as:

$$
\begin{aligned}
\dot{x} & =a(x-y)-y z+w \\
\dot{y} & =-b y+x z \\
\dot{z} & =-c z+d x+x y \\
\dot{w} & =-e(x+y)
\end{aligned}
$$

where $x, y, z, w$ are the state variables and the values of the constants $a, b, c, d$ and $e$ are respectively $0.98,9.00$, $50.00,0.06$ and 0.90 . The circuit components of the hyperchatic system consists of adder, inverter, integrator and multiplier as shown in Fig. 1. The differential equation from the circuit is as follows

$$
\begin{aligned}
& \frac{d x}{d \tau}=\frac{1}{R C}\left(\frac{R}{R_{1}} x-\frac{R}{10 R_{3}} y z+\frac{R}{R_{4}} w\right) \\
& \frac{d y}{d \tau}=\frac{1}{R C}\left(-\frac{R}{R_{5}} y+\frac{R}{10 R_{6}} x z\right) \\
& \frac{d z}{d \tau}=\frac{1}{R C}\left(-\frac{R}{R_{7}} z+\frac{R}{R_{8}} x+\frac{R}{10 R_{9}} x y\right) \\
& \frac{d w}{d \tau}=\frac{1}{R C}\left(-\frac{R}{R_{10}} x-\frac{R}{R_{11}} y\right)
\end{aligned}
$$

If time scale transformation $\tau=10 R C t$ and considering the variable compression $x \rightarrow 0.1 x, y \rightarrow 0.1 y$ and $z \rightarrow 0.1 z$ on Eqn.2, taking $R_{1}=R_{2}$ and $R_{10}=R_{11}$ then we have:

$$
\begin{aligned}
\frac{d x}{d \tau} & =10 \frac{R}{R_{1}} x-y-\frac{R}{10 R_{3}} y z+10 \frac{R}{R_{4}} w \\
\frac{d y}{d \tau} & =-10 \frac{R}{R_{5}} y+\frac{R}{10 R_{6}} x z \\
\frac{d z}{d \tau} & =-\frac{R}{R_{7}} z+10 \frac{R}{R_{8}} x+\frac{R}{10 R_{9}} x y \\
\frac{d w}{d \tau} & =-10 \frac{R}{R_{10}} x-10 \frac{R}{R_{11}} y
\end{aligned}
$$

Comparing Eqn.(1) with Eqn. (3), we have: $a=$ $10 \frac{R}{R_{1}}, b=10 \frac{R}{R_{5}}, c=10 \frac{R}{R_{7}}, d=10 \frac{R}{R_{8}}$ $e=10 \frac{R}{R_{10}}$, where $R=R_{4}=R_{5}=R_{6}=R_{7}=$ $R_{10}=R_{11}=R_{12}=R_{13}=R_{17}=R_{18}=R_{19}=$ $R_{20}=R_{22}=R_{23}=10 \mathrm{k} \Omega, R_{1}=R_{2}=R_{14}=R_{16}=$ $10 k \Omega, R_{3}=102 k \Omega, R_{9}=1.1 k \Omega, R_{1} 5=1.67 M \Omega$ and $R_{21}=111 k \Omega$. Capacitors $\left(C_{1}\right)-\left(C_{4}\right)=10 n F$

The analog circuit is shown in Fig. 1 and phase portraits of the hyperchaotic attractor obtained via MultiSIM and analog circuit are shown in Fig. 2

\section{Theory of Cyclic Coupling}

The cyclic coupling process involves engagement of a pair of variable of one system with a different pair of variable from another system in a bidirectional manner. 


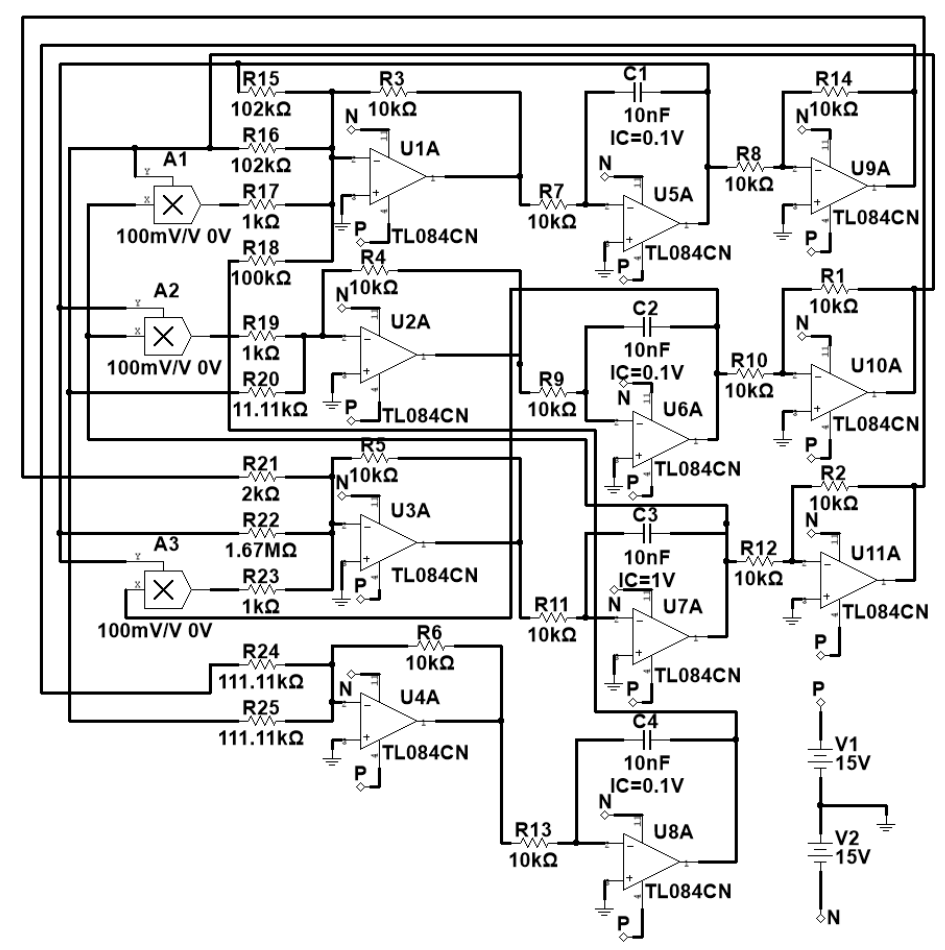

Figure 1. Analog circuit of 4D hyperchaotic system with corresponding power supply
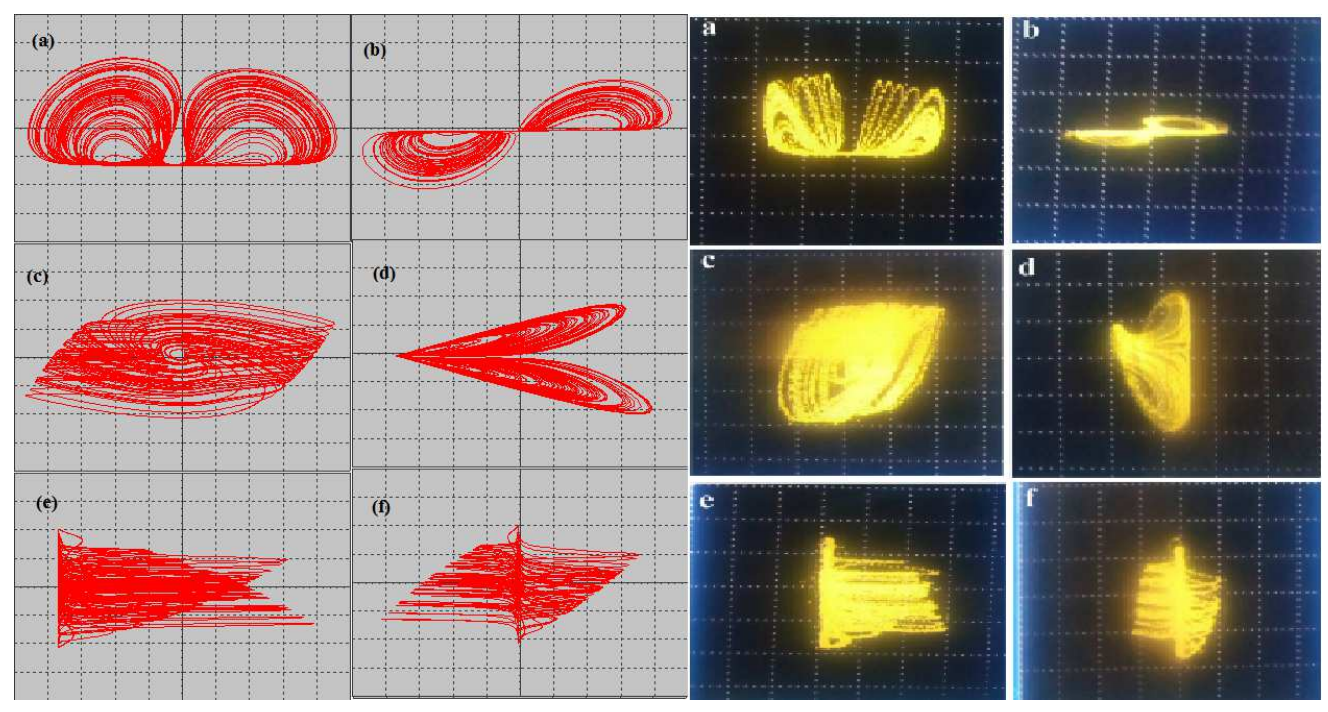

Figure 2. Two dimensional phase portraits of the hyperchaotic attractor for (a) $x-y$, (b) $x-z$, (c) $x-w$, (d) $y-z$, (e) $y-w$ and (f) $z-w$ axes obtained through MultiSIM software (left hand side) and analog circuit (right hand side)

Consider cyclic coupling for two systems each of $n$ dimension. The two systems are:

$$
\begin{gathered}
\dot{x}=B x+f(x)+\beta_{i} H_{i, j}(x, y) \\
\dot{y}=B y+g(y)+\beta_{j} H_{i, j}(x, y)
\end{gathered}
$$

where $x=\left(x_{1}, x_{2}, x_{3}, \ldots x_{n}\right)^{T}, y=\left(y_{1}, y_{2}, y_{3}, \ldots y_{n}\right)^{T}$ are the dynamical state variables of the systems. Matrix $A$ represents the linear part of the system. $f$ and $g$ are non linear part of the system. $\beta_{i}, \beta_{j}(1, j=1,2,3, \ldots n)$ are coupling parameters, where $\beta_{i}$ is the coupling parameter on the first system and $\beta_{j}$ is the coupling parameter on the second system. $H_{i, j}(x, y)$ is the output matrix of each pair of the system that are involved in the cyclic coupling process.

For better understanding, we consider cyclic cou- 
pling using the following pair of systems. In this example, we shall use $x_{1}, x_{2}, x_{3}, \ldots x_{n}$ for the dynamical variables of the first system and $y_{1}, y_{2}, y_{3}, \ldots y_{n}$ for the dynamical variables of the second system. Now we look at possible independent coupling choices for systems of different dimensions: (a) cyclic coupling between two systems of two dimensions each gives only one independent coupling choice $x_{1} \rightarrow y_{1}$ and $x_{2} \leftarrow y_{2}$; (b) cyclic coupling between two systems of three dimensions each gives three independent coupling choices (i) $x_{1} \rightarrow y_{1}$ and $x_{2} \leftarrow y_{2}$ (ii) $x_{1} \rightarrow y_{1}$ and $x_{3} \leftarrow y_{3}$ (iii) $x_{2} \rightarrow y_{2}$ and $x_{3} \leftarrow y_{3}$; (c) cyclic coupling between two systems of four dimensions each gives six independent coupling choices (i) $x_{1} \rightarrow y_{1}$ and $x_{2} \leftarrow y_{2}$ (ii) $x_{1} \rightarrow y_{1}$ and $x_{3} \leftarrow y_{3}$ (iii) $x_{1} \rightarrow y_{1}$ and $x_{4} \leftarrow y_{4}$ (iv) $x_{2} \rightarrow y_{2}$ and $x_{3} \leftarrow y_{3}$ (v) $x_{2} \rightarrow y_{2}$ and $x_{4} \leftarrow y_{4}$ (vi) $x_{3} \rightarrow y_{3}$ and $x_{4} \leftarrow y_{4}$. We noticed that the number of independent choices for cyclic coupling between two $n$ dimension system is ${ }^{n} C_{2}$. For example: $n=2$, gives 1 independent choice; $n=3$ gives 3 independent choices; $n=4$ gives 6 independent choices; $n=5$ gives 10 independent choices and $n=n$ gives ${ }^{n} C_{2}=n(n-1) / 2$ ! independent choices.

In order to establish the stability criteria and the coupling strength threshold for synchronization of cyclic coupled systems (4) is written such that $e_{1}$ and $e_{2}$ are are taken as deviations of the system from synchronized state. Then the variation equation of the deviation of $e_{1}$ and $e_{2}$ can be written as

$$
\begin{aligned}
& \dot{e_{1}}=B e_{1}+f^{\prime}(x) e_{1}+\beta_{i} H_{i, j}\left(e_{2}-e_{1}\right) \\
& \dot{e_{2}}=B e_{2}+g^{\prime}(y) e_{2}+\beta_{j} H_{i, j}\left(e_{1}-e_{2}\right)
\end{aligned}
$$

where $B$ is a linear matrix, $f^{\prime}(x)$ and $g^{\prime}(y)$ are non linear functions. Using the approximation in [Khan and Poria, 2012], the time average of $f^{\prime}(x)$ and $g^{\prime}(y)$ is denoted by $\alpha$. Now, synchronization error is defined as

$$
e=e_{2}-e_{1}
$$

Substitution of (5) into time derivative of (6) yields

$$
\dot{e}=\left(B+\alpha I-\beta_{i} H_{i, j}-\beta_{j} H_{i, j}\right) e
$$

the error dynamics is stable if

$$
P=B+\alpha I-\beta_{i} H_{i, j}-\beta_{j} H_{i, j}<0
$$

otherwise, it is not stable. Therefore, $P$ is a significant equation in determination of the stability of cyclic coupled systems. Moreover, the stability criteria for complete synchronization are derived from the negative real parts of the eigenvalues $\lambda$ of $P$ according to RouthHurwitz stability criterion. Also, $\beta_{i}, \beta_{j}$ and $\alpha$ are real value and non-negative.

\section{Derivation of Synchronization Criteria}

This section deals with derivation of analytical criteria for the cyclic coupled hyperchaotic system. The cyclic coupling requires that two pairs of variables are engaged in the coupling such that there are twelve possible topologies of cyclic coupling for two four dimensional systems, out of which six are independent while the other six topologies are symmetric for identical oscillators.

Now, considering 2 pairs of variables for the 4-dimensional systems, 6 independent options are;

(i) $x_{1} \rightarrow x_{2}, y_{1} \leftarrow y_{2}$ when $H_{1,2}=\operatorname{diag}(1,1,0,0)$

(ii) $x_{1} \rightarrow x_{2}, z_{1} \leftarrow z_{2}$ when $H_{1,3}=\operatorname{diag}(1,0,1,0)$

(iii) $x_{1} \rightarrow x_{2}, w_{1} \leftarrow w_{2}$ when $H_{1,4}=\operatorname{diag}(1,0,0,1)$

(iv) $y_{1} \rightarrow y_{2}, z_{1} \leftarrow z_{2}$ when $H_{2,3}=\operatorname{diag}(0,1,1,0)$

(v) $y_{1} \rightarrow y_{2}, w_{1} \leftarrow w_{2}$ when $H_{2,4}=\operatorname{diag}(0,1,0,1)$

(vi) $z_{1} \rightarrow z_{2}, w_{1} \leftarrow w_{2}$ when $H_{3,4}=\operatorname{diag}(0,0,1,1)$

The arrows in the equations above indicate the direction of coupling. To establish the concept of cyclic coupling we use the stability criteria in (8)

\subsection{Cyclic Coupling Between $x$ and $y$ Variables}

When $H_{1,2}=\operatorname{diag}(1,1,0,0)$, we obtain

$$
\begin{aligned}
\dot{x_{1}} & =a\left(x_{1}-y_{1}\right)-y_{1} z_{1}+w_{1} \\
\dot{y_{1}} & =-b y_{1}+x_{1} z_{1}+\beta_{2}\left(y_{2}-y_{1}\right) \\
\dot{z_{1}} & =-c z_{1}+d x_{1}+x_{1} y_{1} \\
\dot{w_{1}} & =-e\left(x_{1}+y_{1}\right) \\
\dot{x_{2}} & =a\left(x_{2}-y_{2}\right)-y_{2} z_{2}+w_{2}+\beta_{1}\left(x_{1}-x_{2}\right) \\
\dot{y_{2}} & =-b y_{2}+x_{2} z_{2} \\
\dot{z_{2}} & =-c z_{2}+d x_{2}+x_{2} y_{2} \\
\dot{w_{2}} & =-e\left(x_{2}+y_{2}\right)
\end{aligned}
$$

Using the stability condition (8) in (9), we have

$P=\left(\begin{array}{cccc}a & -a & 0 & 1 \\ 0 & -b & 0 & 0 \\ d & 0 & -c & 0 \\ -e & -e & 0 & 0\end{array}\right)+\left(\begin{array}{cccc}\alpha & 0 & 0 & 0 \\ 0 & \alpha & 0 & 0 \\ 0 & 0 & \alpha & 0 \\ 0 & 0 & 0 & \alpha\end{array}\right)$ -

$\left(\begin{array}{cccc}\beta_{1} & 0 & 0 & 0 \\ 0 & \beta_{2} & 0 & 0 \\ 0 & 0 & 0 & 0 \\ 0 & 0 & 0 & 0\end{array}\right)$

$$
P=\left(\begin{array}{cccc}
\alpha+a-\beta_{1} & -a & 0 & 1 \\
0 & \alpha-b-\beta_{2} & 0 & 0 \\
d & 0 & \alpha-c & 0 \\
-e & -e & 0 & \alpha
\end{array}\right)
$$

The eigenvalues of matrix $P$ are,

$\lambda_{1}=\alpha-c$

$\lambda_{2}=\alpha-b-\beta_{2}$

$\lambda_{3,4}=\frac{-\left(\beta_{1}-a-2 \alpha\right) \pm \sqrt{\left(\beta_{1}-a-2 \alpha\right)^{2}-4\left[\alpha^{2}+\left(a-\beta_{1}\right) \alpha-e\right]}}{2}$

So that the required stability conditions are:

(1) For $\lambda_{1}=\alpha-c$, we have $\alpha<c$ 
(2) For $\lambda_{2}=\alpha-b-\beta_{2}$, we have $\beta_{2}>\alpha-b$

(3) If $\left(\beta_{1}+\beta_{2}-a-2 \alpha\right)^{2}<4\left[\left(\alpha-\beta_{2}\right)\left(\alpha-\beta_{1}+a\right)-e\right]$, then $\lambda_{3,4}$ are complex and stability condition is $\beta_{1}+\beta_{2}>(2 \alpha+a)$

(4) If $\left(\beta_{1}+\beta_{2}-a-2 \alpha\right)^{2}>4\left[\left(\alpha-\beta_{2}\right)\left(\alpha-\beta_{1}+a\right)-e\right]$, then $\lambda_{3,4}$ are real and stability condition is $\beta_{1}+\beta_{2}>(2 \alpha+a)$ and $\left[\left(\alpha-\beta_{2}\right)\left(\alpha-\beta_{1}+a\right)-e\right]>0$

Choosing a suitable value of $\beta_{1}=1.1$ and $\beta_{2}=2.98$, complete synchronization was obtained.

\subsection{Cyclic Coupling Between $x$ and $z$ Variables}

When $H_{1,3}=\operatorname{diag}(1,0,1,0)$, we obtain

$$
\begin{aligned}
\dot{x_{1}} & =a\left(x_{1}-y_{1}\right)-y_{1} z_{1}+w_{1} \\
\dot{y_{1}} & =-b y_{1}+x_{1} z_{1} \\
\dot{z_{1}} & =-c z_{1}+d x_{1}+x_{1} y_{1}+\beta_{2}\left(z_{2}-z_{1}\right) \\
\dot{w_{1}} & =-e\left(x_{1}+y_{1}\right) \\
\dot{x_{2}} & =a\left(x_{2}-y_{2}\right)-y_{2} z_{2}+w_{2}+\beta_{1}\left(x_{1}-x_{2}\right) \\
\dot{y_{2}} & =-b y_{2}+x_{2} z_{2} \\
\dot{z_{2}} & =-c z_{2}+d x_{2}+x_{2} y_{2} \\
\dot{w_{2}} & =-e\left(x_{2}+y_{2}\right)
\end{aligned}
$$

Using the stability condition (8) in (10), we have

$$
P=\left(\begin{array}{cccc}
a & -a & 0 & 1 \\
0 & -b & 0 & 0 \\
d & 0 & -c & 0 \\
-e & -e & 0 & 0
\end{array}\right)+\left(\begin{array}{cccc}
\alpha & 0 & 0 & 0 \\
0 & \alpha & 0 & 0 \\
0 & 0 & \alpha & 0 \\
0 & 0 & 0 & \alpha
\end{array}\right) \text { - }
$$$$
\left(\begin{array}{cccc}
\beta_{1} & 0 & 0 & 0 \\
0 & 0 & 0 & 0 \\
0 & 0 & \beta_{2} & 0 \\
0 & 0 & 0 & 0
\end{array}\right)
$$

$$
P=\left(\begin{array}{cccc}
\alpha+a-\beta_{1} & -a & 0 & 1 \\
0 & \alpha-b & 0 & 0 \\
d & 0 & \alpha-c-\beta_{2} & 0 \\
-e & -e & 0 & \alpha
\end{array}\right)
$$

The eigenvalues of matrix $P$ are,

$$
\begin{aligned}
& \lambda_{1}=\alpha-c-\beta_{2} \\
& \lambda_{2}=\alpha-b \\
& \lambda_{3,4}=\frac{-\left(\beta_{1}-a-2 \alpha\right) \pm \sqrt{\left(\beta_{1}-a-2 \alpha\right)^{2}-4\left[\left(\alpha-\beta_{2}\right)\left(\alpha-\beta_{1}+a\right)-e\right]}}{2}
\end{aligned}
$$

So that the required stability conditions are:

(1) For $\lambda_{1}=\alpha-c$, we have $\alpha<c$

(2) For $\lambda_{2}=\alpha-b$, we have $\alpha<b$

(3) If $\left(\beta_{1}-a-2 \alpha\right)^{2}<4\left[\alpha^{2}+\left(a-\beta_{1}\right) \alpha-e\right]$, then $\lambda_{3,4}$ are complex and stability condition is $\beta_{1}>(2 \alpha+a)$

(4) If $\left(\beta_{1}-a-2 \alpha\right)^{2}>4\left[\alpha^{2}+\left(a-\beta_{1}\right) \alpha-e\right]$, then $\lambda_{3,4}$ are real and stability condition is $\beta_{1}>(2 \alpha+a)$ and $\left[\alpha^{2}+\left(a-\beta_{1}\right) \alpha-e\right]>0$
Choosing a suitable value of $\beta_{1}=1.1$ and $\beta_{2}=2.98$, complete synchronization was obtained.

\subsection{Cyclic Coupling Between $x$ and $w$ Variables}

When $H_{1,4}=\operatorname{diag}(1,0,0,1)$, we obtain

$$
\begin{aligned}
\dot{x_{1}} & =a\left(x_{1}-y_{1}\right)-y_{1} z_{1}+w_{1} \\
\dot{y_{1}} & =-b y_{1}+x_{1} z_{1} \\
\dot{z_{1}} & =-c z_{1}+d x_{1}+x_{1} y_{1} \\
\dot{w_{1}} & =-e\left(x_{1}+y_{1}\right)+\beta_{2}\left(w_{2}-w_{1}\right) \\
\dot{x_{2}} & =a\left(x_{2}-y_{2}\right)-y_{2} z_{2}+w_{2}+\beta_{1}\left(x_{1}-x_{2}\right) \\
\dot{y_{2}} & =-b y_{2}+x_{2} z_{2} \\
\dot{z_{2}} & =-c z_{2}+d x_{2}+x_{2} y_{2} \\
\dot{w_{2}} & =-e\left(x_{2}+y_{2}\right)
\end{aligned}
$$

Using the stability condition (8) in (11), we have

$$
\begin{aligned}
& P=\left(\begin{array}{cccc}
a & -a & 0 & 1 \\
0 & -b & 0 & 0 \\
d & 0 & -c & 0 \\
-e & -e & 0 & 0
\end{array}\right)+\left(\begin{array}{cccc}
\alpha & 0 & 0 & 0 \\
0 & \alpha & 0 & 0 \\
0 & 0 & \alpha & 0 \\
0 & 0 & 0 & \alpha
\end{array}\right)- \\
& \left(\begin{array}{cccc}
\beta_{1} & 0 & 0 & 0 \\
0 & 0 & 0 & 0 \\
0 & 0 & 0 & 0 \\
0 & 0 & 0 & \beta_{2}
\end{array}\right)
\end{aligned}
$$$$
P=\left(\begin{array}{cccc}
\alpha+a-\beta_{1} & -a & 0 & 1 \\
0 & \alpha-b & 0 & 0 \\
d & 0 & \alpha-c & 0 \\
-e & -e & 0 & \alpha-\beta_{2}
\end{array}\right)
$$

The eigenvalues of matrix $P$ are,

$\lambda_{1}=\alpha-c-$

$\lambda_{2}=\alpha-b$

$\lambda_{3,4}=\frac{-\left(\beta_{1}+\beta_{2}-a-2 \alpha\right) \pm \sqrt{\left(\beta_{1}+\beta_{2}-a-2 \alpha\right)^{2}-4\left[\alpha^{2}+\left(a-\beta_{2}\right) \alpha-e\right]}}{2}$

So that the required stability conditions are:

(1) For $\lambda_{1}=\alpha-c-\beta_{2}$, we have $\beta_{2}<\alpha-c$

(2) For $\lambda_{2}=\alpha-b$, we have $\alpha<b$

(3) If $\left(\beta_{1}+\beta_{2}-a-2 \alpha\right)^{2}<4\left[\left(\alpha-\beta_{2}\right)\left(\alpha-\beta_{1}+a\right)-e\right]$, then $\lambda_{3,4}$ are complex and stability condition is $\beta_{1}>(2 \alpha+a)$

(4) If $\left(\beta_{1}+\beta_{2}-a-2 \alpha\right)^{2}>4\left[\left(\alpha-\beta_{2}\right)\left(\alpha-\beta_{1}+a\right)-e\right]$, then $\lambda_{3,4}$ are real and stability condition is $\beta_{1}>(2 \alpha+a)$ and $\left[\left(\alpha^{2}+\left(a-\beta_{1}\right) \alpha-e\right]>0\right.$

Choosing a suitable value of $\beta_{1}=20$ and $\beta_{2}=20$, complete synchronization was obtained.

\subsection{Cyclic Coupling Between $y$ and $z$ Variables}

When $H_{2,3}=\operatorname{diag}(0,1,1,0)$, we obtain 


$$
\begin{aligned}
\dot{x_{1}} & =a\left(x_{1}-y_{1}\right)-y_{1} z_{1}+w_{1} \\
\dot{y_{1}} & =-b y_{1}+x_{1} z_{1} \\
\dot{z_{1}} & =-c z_{1}+d x_{1}+x_{1} y_{1}+\beta_{2}\left(z_{2}-z_{1}\right) \\
\dot{w_{1}} & =-e\left(x_{1}+y_{1}\right) \\
\dot{x_{2}} & =a\left(x_{2}-y_{2}\right)-y_{2} z_{2}+w_{2} \\
\dot{y_{2}} & =-b y_{2}+x_{2} z_{2}+\beta_{1}\left(y_{1}-y_{2}\right) \\
\dot{z_{2}} & =-c z_{2}+d x_{2}+x_{2} y_{2} \\
\dot{w_{2}} & =-e\left(x_{2}+y_{2}\right)
\end{aligned}
$$

Using the stability condition (8) in (12), we have

$$
P=\left(\begin{array}{cccc}
a & -a & 0 & 1 \\
0 & -b & 0 & 0 \\
d & 0 & -c & 0 \\
-e & -e & 0 & 0
\end{array}\right)+\left(\begin{array}{cccc}
\alpha & 0 & 0 & 0 \\
0 & \alpha & 0 & 0 \\
0 & 0 & \alpha & 0 \\
0 & 0 & 0 & \alpha
\end{array}\right)-
$$$$
\left(\begin{array}{cccc}
0 & 0 & 0 & 0 \\
0 & \beta_{1} & 0 & 0 \\
0 & 0 & 0 & 0 \\
0 & 0 & \beta_{2} & 0
\end{array}\right)
$$$$
P=\left(\begin{array}{cccc}
\alpha+a & -a & 0 & 1 \\
0 & \alpha-b-\beta_{1} & 0 & 0 \\
d & 0 & \alpha-c-\beta_{2} & 0 \\
-e & -e & 0 & \alpha
\end{array}\right)
$$

Using the stability condition (8) in (13), we have

$$
\begin{aligned}
& P=\left(\begin{array}{cccc}
a & -a & 0 & 1 \\
0 & -b & 0 & 0 \\
d & 0 & -c & 0 \\
-e & -e & 0 & 0
\end{array}\right)+\left(\begin{array}{llll}
\alpha & 0 & 0 & 0 \\
0 & \alpha & 0 & 0 \\
0 & 0 & \alpha & 0 \\
0 & 0 & 0 & \alpha
\end{array}\right)- \\
& \left(\begin{array}{llll}
0 & 0 & 0 & 0 \\
0 & \beta_{1} & 0 & 0 \\
0 & 0 & 0 & 0 \\
0 & 0 & 0 & \beta_{2}
\end{array}\right) \\
& P=\left(\begin{array}{cccc}
\alpha+a & -a & 0 & 1 \\
0 & \alpha-b-\beta_{1} & 0 & 0 \\
d & 0 & \alpha-c & 0 \\
-e & -e & 0 & \alpha-\beta_{2}
\end{array}\right)
\end{aligned}
$$

The eigenvalues of the matrix $P$ are,

$\lambda_{1}=\alpha-b-\beta_{1}$

$\lambda_{2}=\alpha-c$

$\lambda_{3,4}=\frac{\left(\beta_{2}-2 \alpha-a\right) \pm \sqrt{\left(\beta_{2}-2 \alpha-a\right)^{2}-4\left[\alpha^{2}+\left(a-\beta_{2}\right) \alpha-a \beta_{2}+e\right]}}{2}$

So that the required stability conditions are:

(1) For $\lambda_{1}=\alpha-b-\beta_{1}$, we have $\beta_{1}<\alpha-b$

(2) For $\lambda_{2}=\alpha-c$, we have $\alpha<c$

(3) If $\left(\beta_{2}-a-2 \alpha\right)^{2}<4\left[\left(\alpha^{2}+\left(a-\beta_{2}\right) \alpha-a \beta_{2}+e\right]\right.$, then $\lambda_{3,4}$ are complex and stability condition is $\beta_{2}>2 \alpha+a$ (4) If $\left(\beta_{2}-a-2 \alpha\right)^{2}>4\left[\left(\alpha^{2}+\left(a-\beta_{2}\right) \alpha-a \beta_{2}+e\right]\right.$, then $\lambda_{3,4}$ are real and stability condition is $\beta_{2}>2 \alpha+a$ and $\left[\left(\alpha^{2}+\left(a-\beta_{2}\right) \alpha-a \beta_{2}+e\right]>0\right.$

Choosing a suitable value of $\beta_{1}=45$ and $\beta_{2}=70$, complete synchronization was obtained.

\subsection{Cyclic Coupling Between $z$ and $w$ Variables}

When $H_{3,4}=\operatorname{diag}(0,0,1,1)$, we obtain

(2) For $\lambda_{2}=\alpha-b-\beta_{1}$, we have $\beta_{1}>\alpha-b$

(3) If $(2 \alpha+a)^{2}<4\left[\left(\alpha^{2}+a-e\right]\right.$, then $\lambda_{3,4}$ are complex and stability condition is $2 \alpha<a$

(4) If $(2 \alpha+a)^{2}>4\left[\left(\alpha^{2}+a-e\right]\right.$, then $\lambda_{3,4}$ are real and stability condition is $2 \alpha<a$ and $\left[\alpha^{2}+a-e\right]>0$

The analytical synchronization criterion obtained shows that synchronization cannot be achieved between $y$ and $z$ variables.

\subsection{Cyclic Coupling Between $y$ and $w$ Variables}

When $H_{2,4}=\operatorname{diag}(0,1,0,1)$, we obtain

$$
\begin{aligned}
\dot{x_{1}} & =a\left(x_{1}-y_{1}\right)-y_{1} z_{1}+w_{1} \\
\dot{y_{1}} & =-b y_{1}+x_{1} z_{1} \\
\dot{z_{1}} & =-c z_{1}+d x_{1}+x_{1} y_{1} \\
\dot{w_{1}} & =-e\left(x_{1}+y_{1}\right)+\beta_{2}\left(w_{2}-w_{1}\right) \\
\dot{x_{2}} & =a\left(x_{2}-y_{2}\right)-y_{2} z_{2}+w_{2} \\
\dot{y_{2}} & =-b y_{2}+x_{2} z_{2}+\beta_{1}\left(y_{1}-y_{2}\right) \\
\dot{z_{2}} & =-c z_{2}+d x_{2}+x_{2} y_{2} \\
\dot{w_{2}} & =-e\left(x_{2}+y_{2}\right)
\end{aligned}
$$

$$
\begin{aligned}
\dot{x_{1}} & =a\left(x_{1}-y_{1}\right)-y_{1} z_{1}+w_{1} \\
\dot{y_{1}} & =-b y_{1}+x_{1} z_{1} \\
\dot{z_{1}} & =-c z_{1}+d x_{1}+x_{1} y_{1} \\
\dot{w_{1}} & =-e\left(x_{1}+y_{1}\right)+\beta_{2}\left(w_{2}-w_{1}\right) \\
\dot{x_{2}} & =a\left(x_{2}-y_{2}\right)-y_{2} z_{2}+w_{2} \\
\dot{y_{2}} & =-b y_{2}+x_{2} z_{2} \\
\dot{z_{2}} & =-c z_{2}+d x_{2}+x_{2} y_{2}+\beta_{1}\left(z_{1}-z_{2}\right) \\
\dot{w_{2}} & =-e\left(x_{2}+y_{2}\right)
\end{aligned}
$$

Using the stability condition (8) in (14), we have

$$
P=\left(\begin{array}{cccc}
a & -a & 0 & 1 \\
0 & -b & 0 & 0 \\
d & 0 & -c & 0 \\
-e & -e & 0 & 0
\end{array}\right)+\left(\begin{array}{cccc}
\alpha & 0 & 0 & 0 \\
0 & \alpha & 0 & 0 \\
0 & 0 & \alpha & 0 \\
0 & 0 & 0 & \alpha
\end{array}\right) \text { - }
$$


$\left(\begin{array}{llll}0 & 0 & 0 & 0 \\ 0 & 0 & 0 & 0 \\ 0 & 0 & \beta_{1} & 0 \\ 0 & 0 & 0 & \beta_{2}\end{array}\right)$
$P=\left(\begin{array}{cccc}\alpha+a & -a & 0 & 1 \\ 0 & \alpha-b & 0 & 0 \\ d & 0 & \alpha-c-\beta_{1} & 0 \\ -e & -e & 0 & \alpha-\beta_{2}\end{array}\right)$

The eigenvalues of matrix $P$ are,

$\lambda_{1}=\alpha-c-\beta_{1}$

$\lambda_{2}=\alpha-b$

$\lambda_{3,4}=\frac{-\left(\beta_{2}-2 \alpha-a\right) \pm \sqrt{\left(\beta_{2}-2 \alpha-a\right)^{2}-4\left[(\alpha+a)\left(\alpha-\beta_{2}\right)-e\right]}}{2}$

So that the required stability conditions are:

(1) For $\lambda_{1}=\alpha-c-\beta_{1}$, we have $\beta_{1}<\alpha-c$

(2) For $\lambda_{2}=\alpha-b$, we have $\alpha<b$

(3) If $\left(\lambda_{2}-a-2 \alpha\right)^{2}<4\left[(\alpha+a)\left(\alpha-\lambda_{2}\right)-e\right]$, then $\lambda_{3,4}$ are complex and stability condition is $\lambda_{2}>2 \alpha+a$ (4) If $\left(\lambda_{2}-a-2 \alpha\right)^{2}>4\left[(\alpha+a)\left(\alpha-\lambda_{2}-e\right]\right.$, then $\lambda_{3,4}$ are real and stability condition is $\lambda_{2}>2 \alpha+a$ and $\left[(\alpha+a)\left(\alpha-\lambda_{2}\right)-e\right]>0$

Choosing a suitable value of $\beta_{1}=1$ and $\beta_{2}=1$, complete synchronization was obtained.

\section{Results and Discussion}

Having established the analytical criterion for synchronization in each pair of the cyclic coupled variables, we shall confirm each of the derived analytical criterion using MultiSIM software and analog circuit. Now, we implement the analytical criterion obtained for each pair of the hyperchaotic system with each of system with different initial conditions $(1,0,1,5)$ and $(5.5,6,7.2,8)$ on MultiSIM software. The results from MultiSIM software shown in Fig 3 confirm the correctness and effectiveness of the derived analytical criterion. The results in Fig. 3 show that five out of the six topology synchronized except topology that involves $y$ and $z$ variables. The results in Fig. 3 is in perfect consonance with the analytical criterion obtained in section 4 . On the other hand, the circuit realization involves integrators and summers built with operational amplifiers, TL084CN $\left(U_{i A}\right)$, multipliers $(A 1-A 6)$, power supply unit, resistors and capacitors. The two analog circuits captured in Fig. 4, with initial conditions $(1,0,1,5)$ and $(5.5,6,7.2,8)$ used for different cyclic synchronization paths. The coupling constant which determine the small or large window of the phase portrait of the attractor can be generated from $k_{x}, k_{y}, k_{z}$ and $k_{w}$ depending on the choice of the trajectory of the cyclic path, where $k_{x}=k\left(x_{1} \rightarrow x_{2}\right)$ or $k\left(x_{1} \leftarrow x_{2}\right), k_{y}=k\left(y_{1} \rightarrow y_{2}\right)$ or $k\left(y_{1} \leftarrow y_{2}\right)$, $k_{z}=k\left(z_{1} \rightarrow z_{2}\right)$ or $k\left(z_{2} \leftarrow z_{1}\right)$ and $k_{w}=k\left(w_{1} \rightarrow w_{2}\right)$ or $k\left(w_{2} \leftarrow w_{1}\right)$. To achieve the desired goal, the coupling constant is varied according to analytical value obtained in section 4 and the results obtained are shown in Fig.
5. The results in depict in Fig. 5 show that synchronization is observed in each of the five pair of the variable considered. The analog circuit implementation of synchronization between cyclic coupling variables $y$ and $z$ variables is not shown in Fig. 5 because the analytical and MultiSIM software simulation already confirm there is no synchronization in $y$ and $z$ topology.

\section{Conclusions}

Cyclic coupling synchronization scheme for two identical 4D hyperchaotic systems has been proposed in this research paper. The results from numerical and experimental simulations confirm the effectiveness and the feasibility of the derived analytical criterion. In other word, the analytical, numerical and analog implementation confirm synchronization in five out of six available topologies. The main advantage of cyclic coupling is that it gives several choices for synchronization, as a result, synchronization choices can be optimized. It help to limit the choice of synchronization to a particular pair of variables or any desired pair of variables that would give the least synchronization time and synchronization cost. Hence, cyclic coupling synchronization scheme can be used to save synchronization time and synchronization cost.

\section{References}

Adegoke, E. A., Ojo, K. S., and Njah, A. N. (2013). Adaptive control and synchronization of new hyperchaotic systems with application to secure communication. Journal of the Nigeria Association of Mathematical Physics, 24 (5), pp. 7-16.

Adelakun, A., Adeiza, O. F., and Oketayo, O. O. (2014). Unidirectional synchronization of two identical jerk oscillators with memristor. Journal of Nigerian Association of Mathematical Physics, 28 (2), pp. 35-42.

Adelakun, A. O., Adekoya, M. A., and Odusote, Y. A. (2018). Experimental study of combination synchronization via cyclic coupling. Nigerian Association of Mathematical Physics, Transaction 5, pp. 193-204.

Adelakun, O., Ogunjo, S., and Fuwape, I. (2017). Dynamics of delayed memristive systems in combination chaotic circuits. In Advances in Memristors, Memristive Devices and Systems, pp. 477-492. Springer.

Alligood, K., Sauer, T., and JA, Y. (1997). An introduction to dynamical systems. Springer-Verlag, New York. Belykh, I., Hasler, M., Lauret, M., and Nijmeijer, H. (2005). Synchronization and graph topology. International Journal of Bifurcation and Chaos, 15(11), pp. 3423-3433.

Bera, B. K., Hens, C., Bhowmick, S. K., Pal, P., and Ghosh, D. (2016). Transition from homogeneous to inhomogeneous steady states in oscillators under cyclic coupling. Physics Letters A, 380 (1-2), pp. 130-134.

Dongmo, E. D., Ojo, K. S., Woafo, P., and Njah, A. N. (2018). Difference synchronization of identical and 
(a)

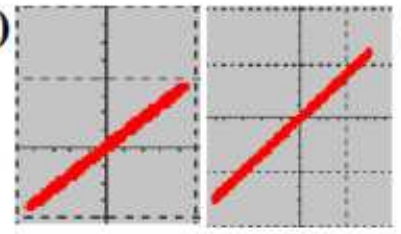

(d)

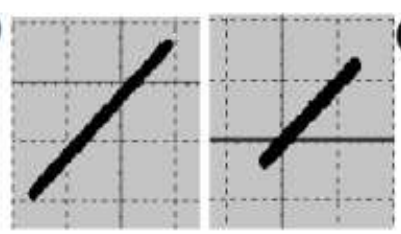

(b)

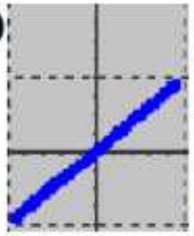

(e)

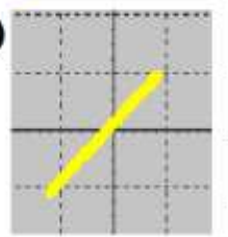

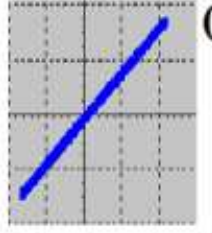
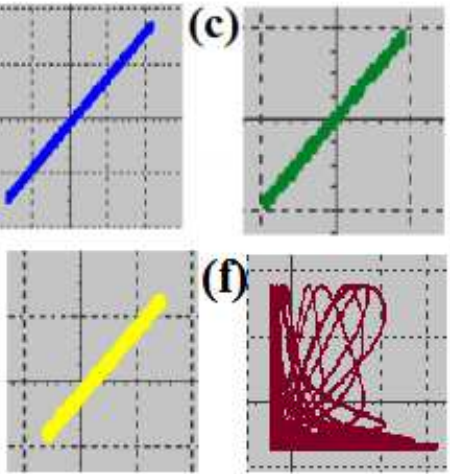

(f)

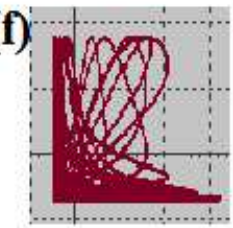

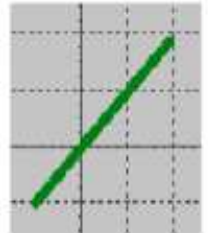

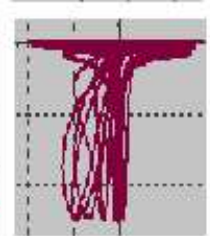

Figure 3. Comfirmation of derived synchronization criterion for the following pair of cyclic coupled variables: (a) $x_{1} \rightarrow x_{2}, y_{1} \leftarrow y_{2}$ (b) $x_{1} \rightarrow x_{2}, z_{1} \leftarrow z_{2}$, (c) $x_{1} \rightarrow x_{2}, w_{1} \leftarrow w_{2}$ (d) $y_{1} \rightarrow y_{2}, w_{1} \leftarrow w_{2}$ and(e) $z_{1} \rightarrow z_{2}, w_{1} \leftarrow w_{2}$ (f) $y_{1} \rightarrow y_{2}, z_{1} \leftarrow z_{2}$ through MultiSIM

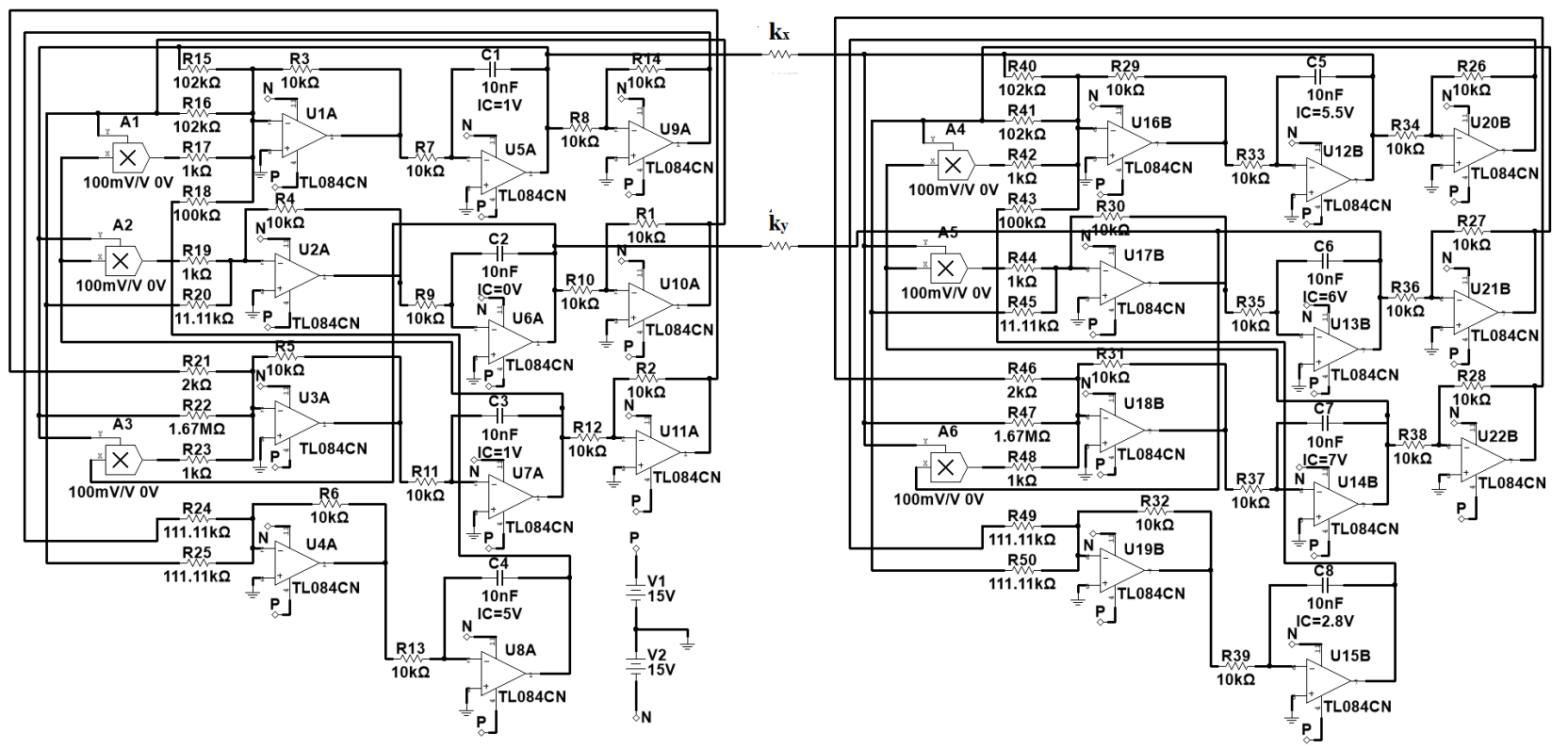

Figure 4. Analog circuit for the cyclic coupled hyperchaotic systems

nonidentical chaotic and hyperchaotic systems of different orders using active backstepping design. Journal of Computational and Nonlinear Dynamics, 13 (5), pp. 051005.

Egunjobi, A., Olusola, O., Njah, A., Saha, S., and Dana, S. (2018). Experimental evidence of chaos synchronization via cyclic coupling. Communications in Nonlinear Science and Numerical Simulation, 56, pp. 588595.

Gasri, A., Ouannas, A., Ojo, K. S., and Pham, V.T. (2018). Coexistence of generalized synchronization and inverse generalized synchronization between chaotic and hyperchaotic systems. Nonlinear Analysis: Modelling and Control, 23 (4), pp. 583-598.

Iqbal, M., Rehan, M., and Hong, K.-S. (2018). Robust adaptive synchronization of ring configured uncertain chaotic fitzhugh-nagumo neurons under directiondependent coupling. Frontiers in neurorobotics, 12, pp. 6.

Kandel, E. R., Schwartz, J. H., Jessell, T. M., of Biochemistry, D., Jessell, M. B. T., Siegelbaum, S., and Hudspeth, A. (2000). Principles of neural science, vol. 4. McGraw-hill New York.

Khan, M. A. and Poria, S. (2012). Projective synchronization of bidirectionally coupled chaotic systems via linear transformations. International Journal of Applied, 1 (4), pp. 541-548.

Kumar, R., Bilal, S., and Ramaswamy, R. (2016). Synchronization properties of coupled chaotic neurons: The role of random shared input. Chaos: An In- 

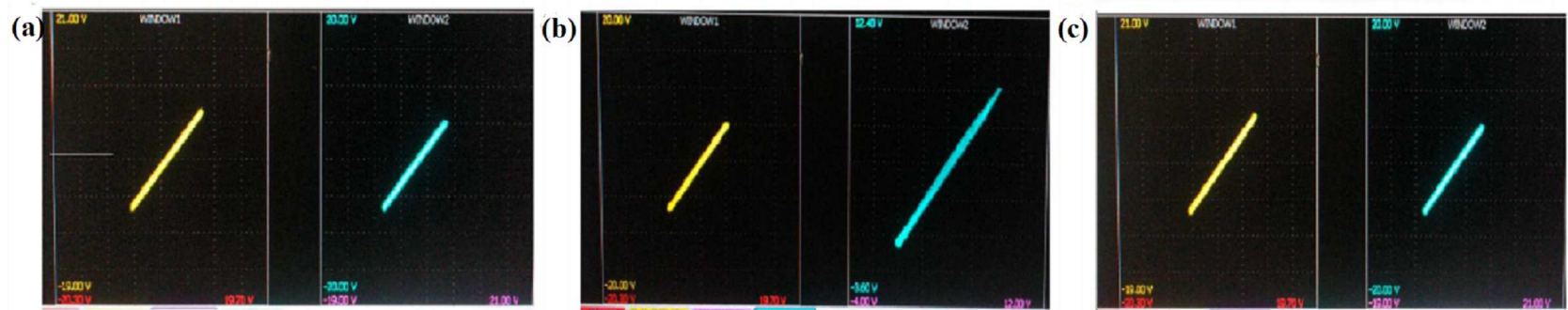

(d)
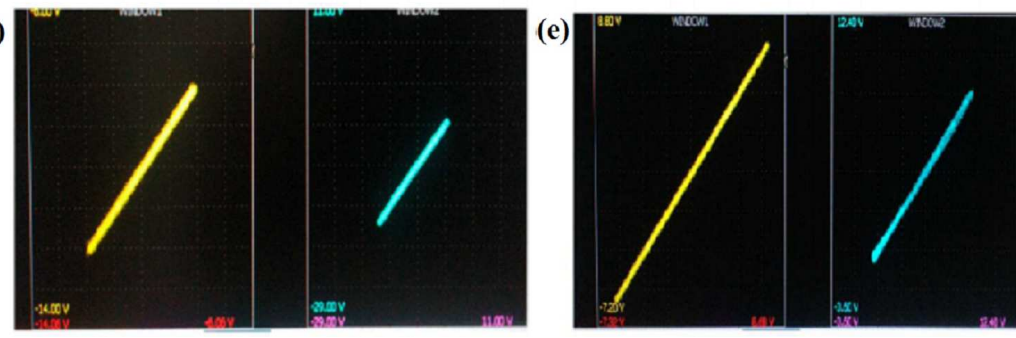

Figure 5. Comfirmation of derived synchronization criterion for the following pair of cyclic coupled variables: (a) $x_{1} \rightarrow x_{2}, y_{1} \leftarrow y_{2}$ (b) $x_{1} \rightarrow x_{2}, z_{1} \leftarrow z_{2}$, (c) $x_{1} \rightarrow x_{2}, w_{1} \leftarrow w_{2}$ (d) $y_{1} \rightarrow y_{2}, w_{1} \leftarrow w_{2}$ and(e) $z_{1} \rightarrow z_{2}, w_{1} \leftarrow w_{2}$ through analog circuit

terdisciplinary Journal of Nonlinear Science, $26(6)$, pp. 063118.

Liu, R., Sun, J. Z., Luo, Y. Q., Sun, W., and Li, W. D. (2011). Research on multi-motor synchronization control based on the ring coupling strategy for cutterhead driving system of shield machines. In Applied Mechanics and Materials, vol. 52, Trans Tech Publ, pp. 65-72.

Lu, J., Ho, D. W., Cao, J., and Kurths, J. (2013). Single impulsive controller for globally exponential synchronization of dynamical networks. Nonlinear Analysis: Real World Applications, 14 (1), pp. 581-593.

Ma, M., Zhou, J., and Cai, J. (2012). Practical synchronization of nonautonomous systems with uncertain parameter mismatch via a single state feedback control. International Journal of Modern Physics C, 23(11), pp. 1250073.

Mengfei, S., Jianxiong, L., and Yiming, F. (2015). Adaptive cross-coupled sliding mode control for synchronization of dual-cylinder electro-hydraulic servo systems. In 2015 34th Chinese Control Conference (CCC), IEEE, pp. 3361-3366.

Njah, A. (2011). Synchronization via active control of parametrically and externally excited $\phi^{6}$ van der pol and duffing oscillators and application to secure communications. Journal of Vibration and control, 17 (4), pp. 493-504.

Njah, A. and Ojo, K. (2010). Synchronization of parametrically and externally excited $\phi^{6}$ van der pol oscillators with application to secure communications. International Journal of Modern Physics B, 24(23), pp. 4581-4893.

Ojo, K., Adeloye, A., and Busari, A. (2016a). Modified function projective synchronization of multistable systems. Ife Journal of Science, 18 (2), pp. 531-540.

Ojo, K., Njah, A., and Ogunjo, S. (2013a). Comparison of backstepping and modified active control in projective synchronization of chaos in an extended bonhöffer-van der pol oscillator. Pramana, 80(5), pp. 825-835.

Ojo, K., Njah, A., and Olusola, O. (2016b). Generalized combination-combination synchronization of chaos in identical orders chaotic systems. J. Appl. Nonlinear Dyn, 5 (1), pp. 43-58.

Ojo, K., Ogunjo, S. T., and Williams, O. (2013b). Mixed tracking and projective synchronization of 5d hyperchaotic system using active control. Cybernetics and Physics, 2(1), pp. 31-36.

Ojo, K., Olusola, O., and Njah, A. (2013c). Chaos synchronization of directionally coupled and periodically modulated josephson junctions and its application to secure communications. The African Review of Physics, 8(0064), pp. 489-498.

Ojo, K. S., Njah, A. N., and Adebayo, G. A. (2011). Anti-synchronization of identical and non-identical van der pol and duffing oscillator with both parametric and external excitations via backstepping approach. International Journal of Modern Physics B, 25(14), pp. 1957-1969.

Okpabi, S. O., Oke, A. A., Akinlami, J., and Opeifa, S. (2017). Backstepping control and synchronization of hyperchaotic lorenz-stenflo system with application to secure communication. Far East Journal of Dynamical Systems, 29 (1), pp. 1-23.

Olusola, O., Njah, A., and Dana, S. (2013). Synchronization in chaotic oscillators by cyclic coupling. The European Physical Journal Special Topics, $222(3-4)$, pp. 927-937.

Ouannas, A. (2014). Synchronization of discrete chaotic systems via double scaling matrix in different dimensions. vol. 3 .

Pecora, L. M. and Carroll, T. L. (1990). Synchroniza- 
tion in chaotic systems. Physical review letters, 64 (8), pp. 821.

Rosenblum, M. G., Pikovsky, A. S., and Kurths, J. (1997). From phase to lag synchronization in coupled chaotic oscillators. Physical Review Letters, 78 (22), pp. 4193.

Rulkov, N. F., Sushchik, M. M., Tsimring, L. S., and Abarbanel, H. D. (1995). Generalized synchronization of chaos in directionally coupled chaotic systems. Physical Review E, 51 (2), pp. 980.

Strogatz, S. H. (1994). Nonlinear dynamics and chaos: with applications to physics, biology, chemistry, and engineering. Addison-Wesley Publishing Company, USA.

Tong, Y.-N., Li, C.-L., and Zhou, F. (2016). Syn- chronization control of single-phase full bridge photovoltaic grid-connected inverter. Optik-International Journal for Light and Electron Optics, 127(4), pp. 1724-1728.

Yang, C.-C. (2012). Robust synchronization and antisynchronization of identical $\phi^{6}$ oscillators via adaptive sliding mode control. Journal of Sound and Vibration, 331 (3), pp. 501-509.

Yu, F., Wang, C., Wan, Q., and Hu, Y. (2013). Complete switched modified function projective synchronization of a five-term chaotic system with uncertain parameters and disturbances. Pramana, 80 (2), pp. 223-235.

Zhang, B. and Deng, F. (2014). Double-compound synchronization of six memristor-based lorenz systems. Nonlinear Dynamics, 77 (4), pp. 1519-1530. 